162

\title{
GRAVIDANZA A TERMINE ED ESPOSIZIONE \\ AL VIRUS VARICELLA ZOSTER : IL RUOLO DEL LABORATORIO DI VIROLOGIA
}

Tenuta R., Savino O., Noto A., Greco F., Giraldi C.

U.O Microbiologia e Virologia

Ospedale Annunziata AO Cosenza

\section{Introduzione}

Il virus della varicella è l'agente eziologico di due comuni patologie virali: la varicella e l'herpes zoster.La varicella in gravidanza può dare esito ad una infezione materno fetale e la gravità della sindrome dipende dall'epoca gestazionale in cui si manifesta l'infezione materna; nella prima metà della gravidanza $\left(0-20^{\circ}\right.$ settimana di gestazione $)$ il rischio di sindrome da varicella congenita è basso $(0,4-2 \%)$. L'insorgenza di varicella nella madre prima o subito dopo il parto può determinare varicella congenita nel $25-50 \%$ dei casi, con una mortalità neonatale del $20-30 \%$.

\section{Materiali e metodi}

Sono venute alla nostra osservazione tre donne in gravidanza tra la $39^{\mathrm{a}}-40^{\mathrm{a}}$ settimana di gestazione, con esposizione familiare al virus. In urgenza è stata valutata la sieroreattività nei confronti di VZV con tecnica di immunofluorescenza indiretta e/o immunoenzimatica.

\section{Risultati}

Due donne presentavano una sieroreattività (presenza di IgG specifiche) nei confronti di VZV, indice di immunita'. Il $3^{\circ}$ caso mostrava suscettibilità (IgG-negative) verso il virus. Questa particolare condizione ha indotto il clinico ad eseguire un parto anticipato dopo solo 12 ore dall'esposizione. Sono state somministrate immunoglobuline specifiche sia alla madre che al neonato.

A distanza di 15 giorni dal parto è stata diagnosticata alla madre, una varicella severa trattata con Acyclovir. La comparsa di una varicella lieve è avvenuta nel neonato a 30 giorni dalla nascita.

Conclusioni

In tutte le donne con gravidanza a termine, esposte al viruse e non consapevoli del proprio stato di suscettibilità, è doveroso eseguire in urgenza, la valutazione delle $\mathrm{IgG}$ specifiche al fine di mettere in atto tutte le procedure terapeutiche, cliniche e di profilassi idonee a prevenire le importanti complicanze della varicella neonatale. 